\title{
Karnak Nord (2020)
}

Les dépôts 2020

Irmgard Hein

\section{OpenEdition}

\section{Journals}

Édition électronique

URL : https://journals.openedition.org/baefe/2814

DOI : $10.4000 /$ baefe. 2814

ISSN : 2732-687X

Éditeur

ResEFE

\section{Référence électronique}

Irmgard Hein, « Karnak Nord (2020) » [notice archéologique], Bulletin archéologique des Écoles françaises à l'étranger [En ligne], Égypte, mis en ligne le 30 mai 2021, consulté le 25 juillet 2021. URL: http://journals.openedition.org/baefe/2814 ; DOI : https://doi.org/10.4000/baefe.2814

Ce document a été généré automatiquement le 25 juillet 2021.

\section{(c)}

Le Bulletin archéologique des Écoles françaises à l'étranger est mise à disposition selon les termes de la Licence Creative Commons Attribution - Pas d'Utilisation Commerciale - Pas de Modification 4.0 International. 


\title{
Karnak Nord (2020)
}

Les dépôts 2020

\author{
Irmgard Hein
}

\section{NOTE DE L'AUTEUR}

Année de la campagne : 2020 ( 3 février - 27 février)

Autorité nationale présente : Merwat Hafes et Mustafa Ala-ed-Din représentaient le Ministère du Tourisme et des Antiquités (MoTA) sous la supervision de Mohamed Yahiyah, Mustafa es-Saghir, Fawzi Helmi Wakil, Ghada Ibrahim Fouad et Ahmed Dowyi (directeur général des magasins de Karnak)

Numéro et intitulé de l'opération de terrain : 17223 - Karnak Nord - Les dépôts et mobilier du trésor de Thoutmosis $\mathrm{I}^{\mathrm{er}}$

Composition de l'équipe de terrain : L'équipe était composée de Irmgard Hein (égyptologue, Universität Wien), Sylvie Marchand (céramologue, Ifao), Gottfried Heumesser (photographe, indépendant), Kristina Hutter (égyptologue, PhD doctorante, Universität Wien), Alfred Hutter (égyptologue, Universität Wien), ainsi que de Lara Borchhardt, Gerda Esche, Alexander Haidegger, Ines Kittinger, Rainer Pauer et Dr. Linda Winkler (étudiants, Universität Wien).

Partenariats institutionnels : Universität Wien, Autriche.

Organismes financeurs : La mission a bénéficié du soutien de Universität Wien (Faculté des sciences historiques et culturelles, Institut d'Égyptologie et VIAS).

\section{Introduction}

1 Nous tenons à remercier explicitement les autorités de Karnak: Mostafa al-Saghir, directeur général de Karnak et de l'avenue des sphinx et Fawzy Helmi Wakil, directeur du temple de Karnak, pour leur soutien. Notre gratitude va également à Madame Ghada Ibrahim Fouad, directrice des missions étrangères à Karnak. Nous souhaitons remercier 
tout particulièrement le directeur général des magasins de Karnak, Ahmed Dowy pour son aide et sa collaboration constructive.

Comme déjà mentionné dans le rapport 2019, à l'été 2019, tous les objets archéologiques provenant des fouilles du Trésor de Thoutmosis I ${ }^{\text {er }}$ ont été déplacés du chantier de Karnak Nord vers le magasin du Cheikh Labib, où nous avons continué le travail de documentation en février 2020. Les travaux se sont déroulés du 3 au 27 février, tous les jours de $7 \mathrm{~h}$ à $15 \mathrm{~h}$, sauf le vendredi.

5 Le matériel archéologique provient des fouilles de l'Ifao à Karnak Nord, menées sous la direction de Jean Jacquet, en 1970-1992. Ces fouilles ont débuté au temple de Harprê dans l'enceinte de Montou. Suite à la découverte du trésor de Thoutmosis $\mathrm{I}^{\mathrm{er}}$, quinze campagnes de fouilles ont été menées afin d'explorer ce complexe situé plus à l'est, en dehors de l'enceinte de Montou. Le bâtiment, du début de la XVIII dynastie, coupe dans les structures des habitations plus anciennes du Moyen Empire et de la Deuxième Période intermédiaire. Par conséquent, des trouvailles plus anciennes (comme des céramiques) étaient également présentes sur le site.

Une construction antérieure au bâtiment du trésor a peut-être existé, mais on ne peut le supposer que sur la base de fragments de décor, un plan de fondation n'ayant pas pu être déterminé ${ }^{1}$.

7 Des modifications ultérieures apportées au bâtiment du trésor pendant l'époque ramesside, son utilisation pendant la Troisième Période intermédiaire ou même plus tard, ont également laissé des traces et du matériel archéologique sur le site. Il en résulte une vaste fourchette chronologique, visible dans l'inventaire puisqu'il couvre une période allant de la $\mathrm{XI}^{\mathrm{e}}$ dynastie jusqu'aux périodes ptolémaïque et romaine.

Les objectifs de la campagne 2020 étaient :

1. Poursuivre la documentation des fragments de reliefs qui constituent les derniers vestiges de la décoration murale originale de l'édifice en calcaire de Thoutmosis $\mathrm{I}^{\mathrm{er}}$.

2. Documenter des fragments de pierre restant dans le dépôt, dont une partie a déjà été publiée.

3. Poursuivre l'enregistrement de l'inventaire des petits objets non céramique qui, à l'exception de quelques pièces individuelles, sont restés inédits jusqu'à présent.

\section{Fragments de relief}

9 En février 2020, nous avons enregistré 2696 fragments de relief, qui étaient stockés dans des paniers; ils proviennent de la décoration originale en calcaire du trésor de Thoutmosis $\mathrm{I}^{\mathrm{er}}$. Toutes les pièces ont été photographiées de face et de dos ${ }^{2}$. Les numéros d'enregistrement, donnés au moment de la fouille, sont souvent conservés, écrits à l'encre noire, au verso. Les fragments sont triés selon les motifs. On y trouve surtout des fragments de frises khekerou, ou des fragments avec des restes de bandes colorées, c'est-à-dire des décorations de bord, de qualité variable. Les fragments avec une 
décoration figurative ont déjà été documentés pendant les années précédentes, en 2018 et 2019.

Dans le volume Karnak-Nord VIII, qui est consacré à la décoration murale du Trésor, Helen Jacquet-Gordon avait déjà pris en compte différents critères dans la reconstitution de la décoration. Le premier critère est l'utilisation de deux matériaux calcaires différents: a) une variante de couleur légèrement grise (proportion plus faible) qui pourrait avoir été utilisée sur les murs extérieurs d'après la position dans laquelle les fragments ont été trouvés, b) une variante blanche à grain fin, qui présente souvent des cassures conchoïdales (concaves-convexes) (voir par exemple fig. 1) et qui est plus fréquente dans les chambres intérieures telles que le sanctuaire de la barque.

11 Le deuxième critère était celui des différentes techniques utilisées, comme le hautrelief ou le relief en creux, critère clairement identifiable ${ }^{3}$.

12 Seuls quelques fragments comportant des éléments de khekerou sont décrits en détail dans les publications précédentes. En 2020, nous avons pu enregistrer un total de 1750 fragments avec ce motif, qui servait de couronnement à une scène, dans différents styles, gravés en relief ou en creux, de manière linéaire. Autant qu'on puisse en juger, des fragments avec des reliefs (fig. 1) peuvent provenir du mur extérieur du sanctuaire. Ceux dont les lignes sont gravées en creux (fig. 2) sont plus fréquents et peuvent être issus des différents murs intérieurs du sanctuaire. Les numéros originaux conservés au verso nous informent sur leur emplacement lors de leur découverte.

Fig. 1. Fragment de relief, frise de khekerou, calcaire, inv. KNUV 20-3342 (photo-no. 2020_P41314, K. Hutter).

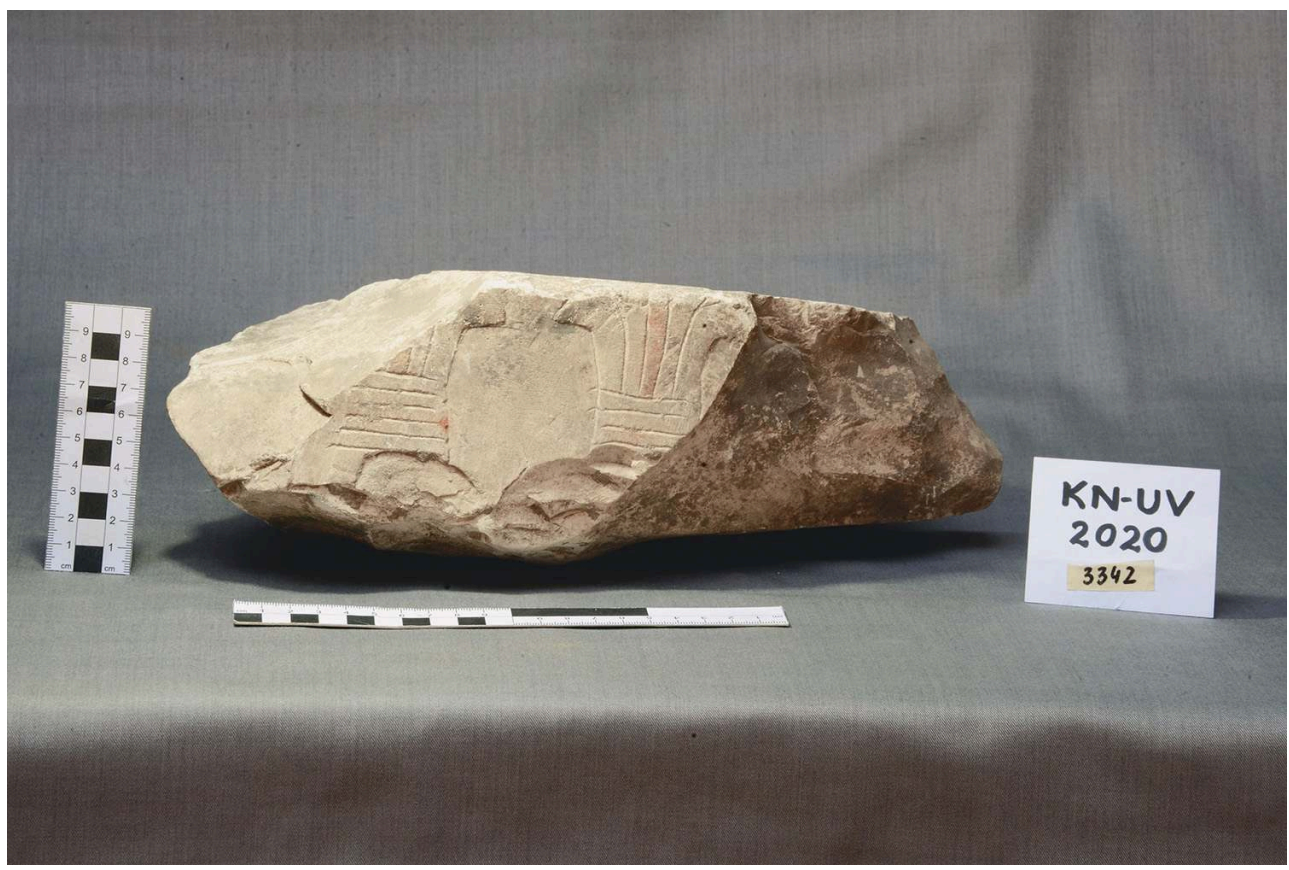

(C) Ifao. 17223_2020_NDMPM_001 
Fig. 2. Fragment de relief, gravé en creux, frise de khekerou, calcaire, inv. KNUV 20-0343 (photono. 2020_P30192, I. Kittinger).

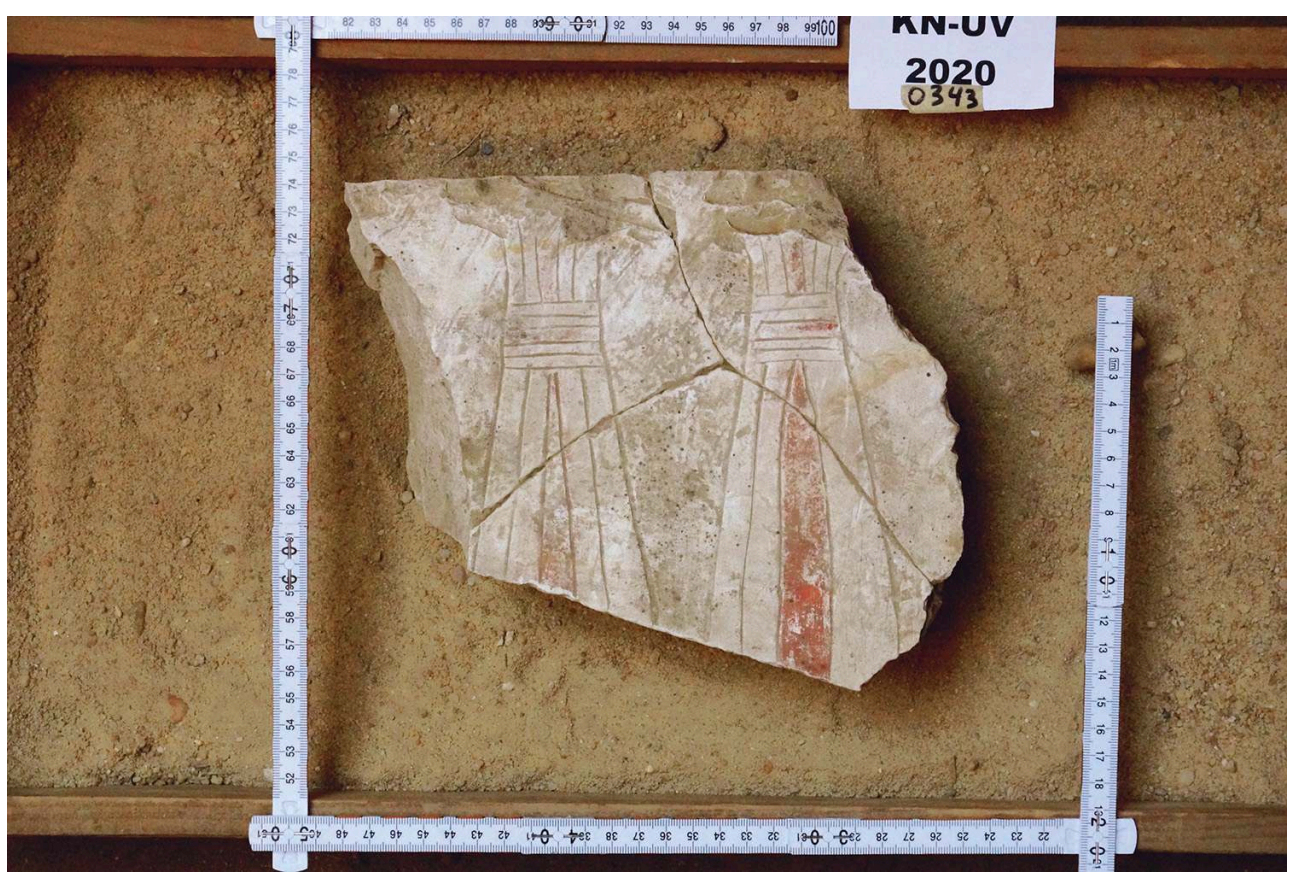

(C) Ifao. 17223_2020_NDMPM_002

Les fragments avec des bordures sont également disponibles dans une large variété de styles et de matériaux. Il s'agit souvent de bordures marginales, qui sont de simples bandes linéaires, ou divisées en rectangles (fig. 3-4). Il y a également des reliefs ou des lignes en creux. Sur certaines pièces, des restes de peinture (rouge, jaune et noir) sont encore conservés. 
Fig. 3. Fragment de relief gravé en creux, bordure divisée en rectangles, calcaire, inv. KNUV 20-0481 (photo-no. 2020_30420, I. Kittinger).

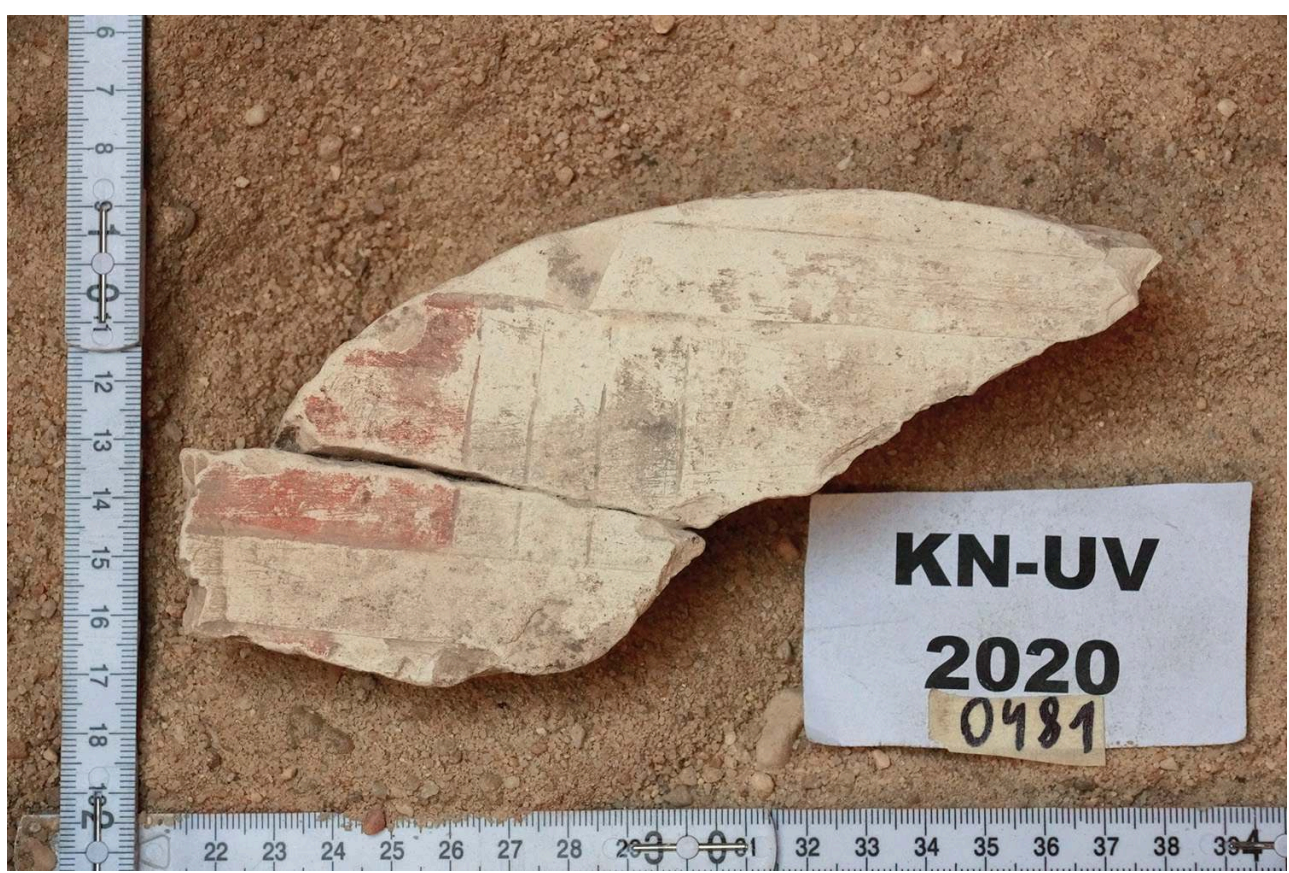

(C) Ifao. 17223_2020_NDMPM_003

Fig. 4. Sept fragments des bordures différentes, en relief et en creux, calcaire, inv. KNUV 20-0367-0373 (photo-no. 2020_30202, G. Esche).

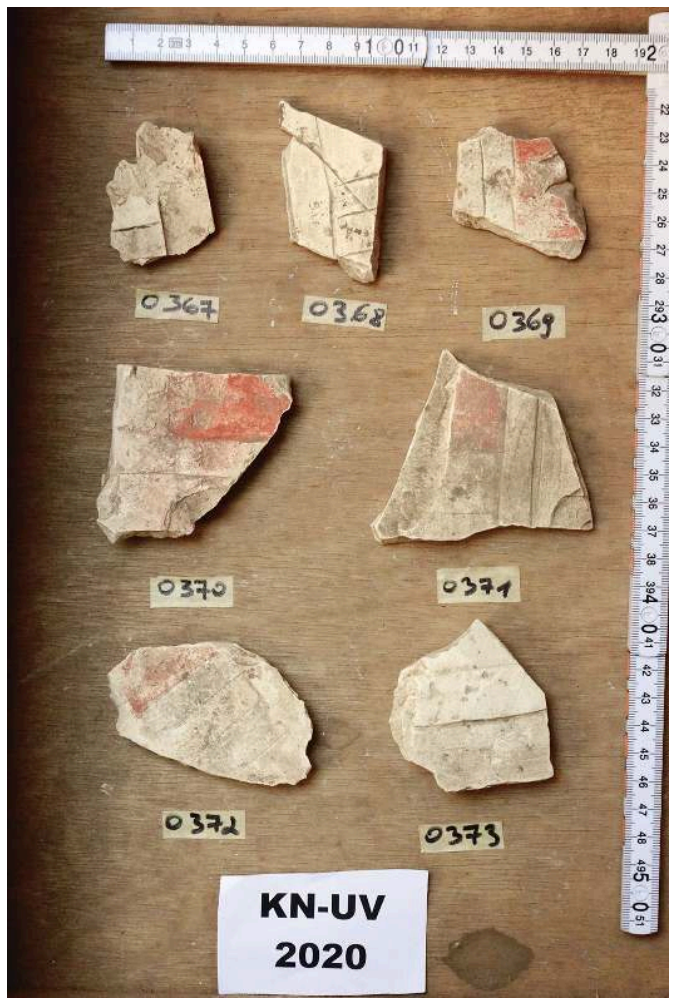

(C) Ifao. 17223_2020_NDMPM_004 


\section{Fragments de pierre} Nord VIII, en grès, il est encore en bon état de conservation, seul le coin supérieur droit à l'avant n'est plus présent. La scène montre une table d'offrande devant laquelle se trouve une princesse avec une boucle de jeunesse et un double collier, qui semble offrir une oie ${ }^{4}$.

Fig. 5. Fragment d'une talatate, grès, inv. KNUV 20-1011 (photo-no. 2020_40048, K. Hutter).

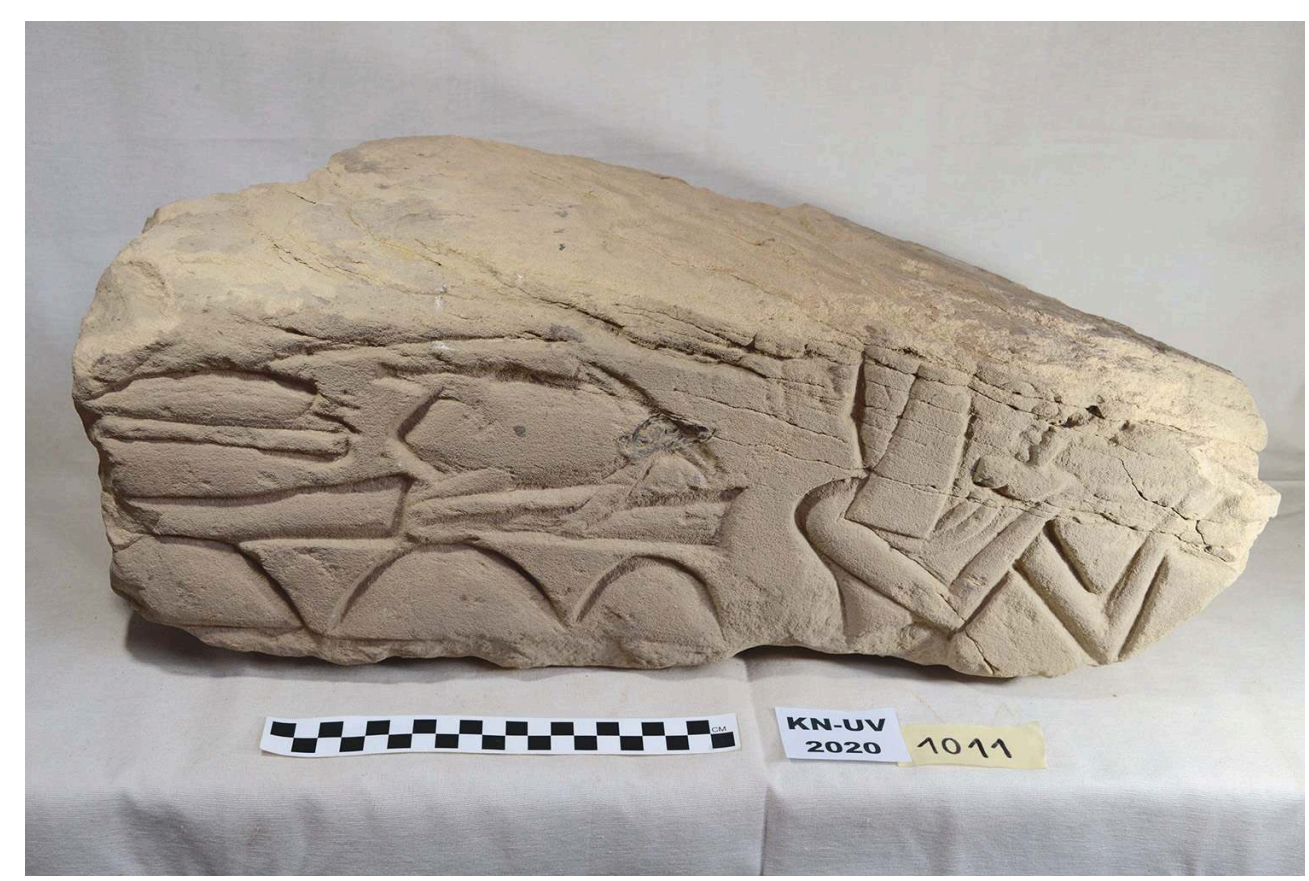

() Ifao. 17223_2020_NDMPM_005

Plusieurs parties de statues en pierre sombre, comme le granit noir ou la granodiorite (ainsi vraisemblablement qu'une roche métasédimentaire), sont également incluses dans l'inventaire des magasins. Parmi celles-ci se trouvent les fragments d'au moins trois statues de Sekhmet inédites. À titre d'exemple, nous montrons ici un fragment de statue debout avec la base d'un piédestal. Il s'agit du pied droit d'une figure portant un bracelet en relief au dessus de la cheville (voir fig. 6). La surface de cette pièce est très bien travaillée, et très bien lissée, alors que d'autres fragments ont une surface laissée rugueuse (non illustrée).

Parmi les exemples non illustrés ici, on peut citer la partie inférieure de la jambe d'une remarquable sculpture à échelle humaine d'un roi debout. On mentionnera également une sculpture incomplète en forme de vache, qui peut être identifiée à un objet déjà illustré par Alexandre Varille ${ }^{5}$. L'étude de ces pièces est en préparation. 
Fig. 6. Fragment d'une statue Sékhmet, granit noir, inv. KNUV 20-1141 (photo-no. 2020_41522, K. Hutter).

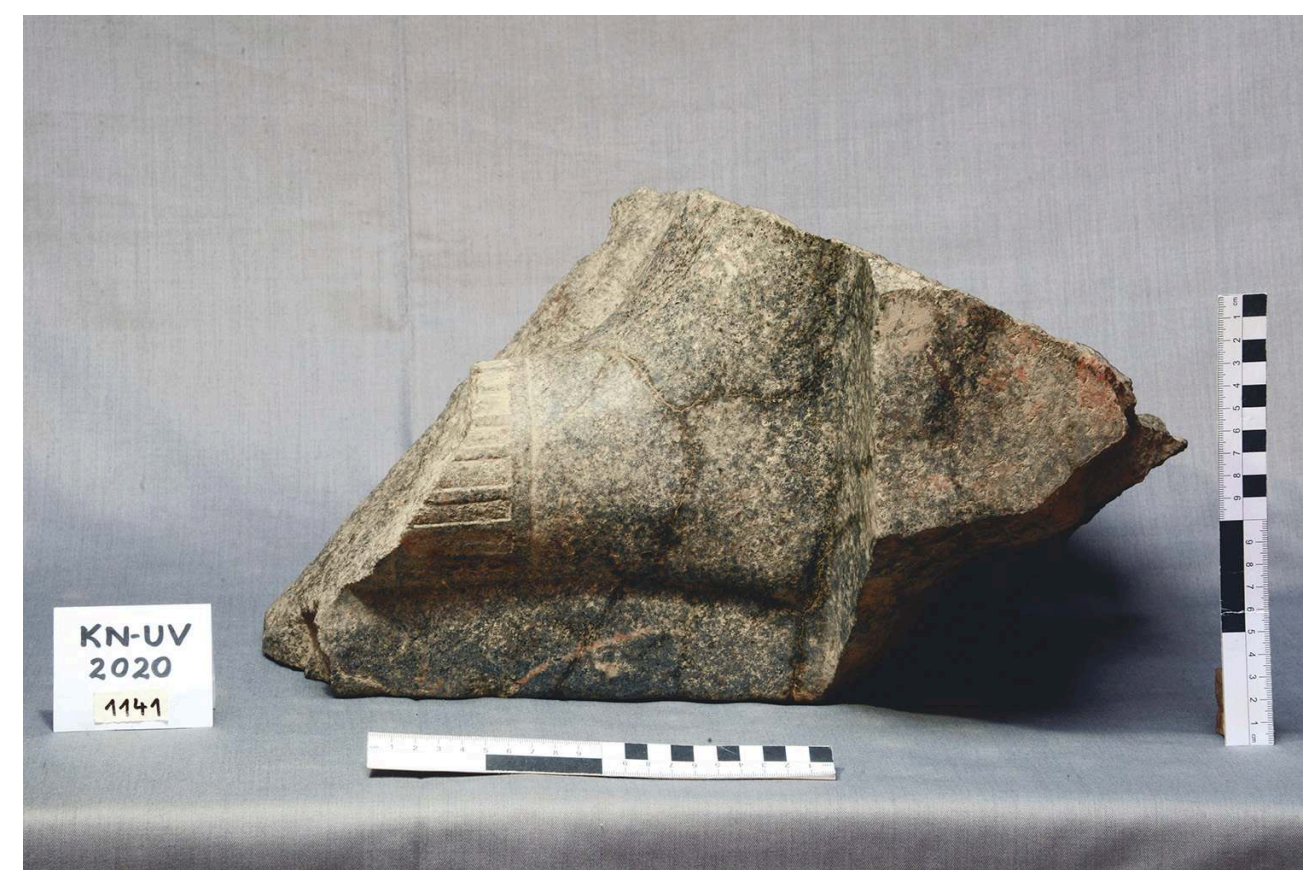

(C) Ifao. 17223_2020_NDMPM_006

18 Au total, 82 fragments de calcaire ont été enregistrés (en dehors des fragments de relief), dont 18 sont des fragments de récipients ou de bassins en pierre grossière. Plusieurs fragments de calcaire peuvent être attribués à un encadrement à tore provenant d'un petit naos. Les inscriptions du cadre latéral du naos ont déjà été reconstituées par H.Jacquet-Gordon, mais les fragments du tore ou sa peinture n'avaient pas été pris en compte ${ }^{6}$. Sur certains fragments du bâton rond, on pouvait reconnaître des traces de peinture noire formant un ruban enroulé qui était disposé en forme de Z. Selon les restes du cartouche conservé dans ces inscriptions, le naos peut être attribué à Thoutmosis II. Il avait probablement été placé dans le magasin $3 \mathrm{du}$ Trésor. 
Fig. 7. Fragment de tore d'un naos de Thoutmosis II, calcaire, inv. KNUV 20-1129-11 (photono. 2020_32145, K. Hutter).

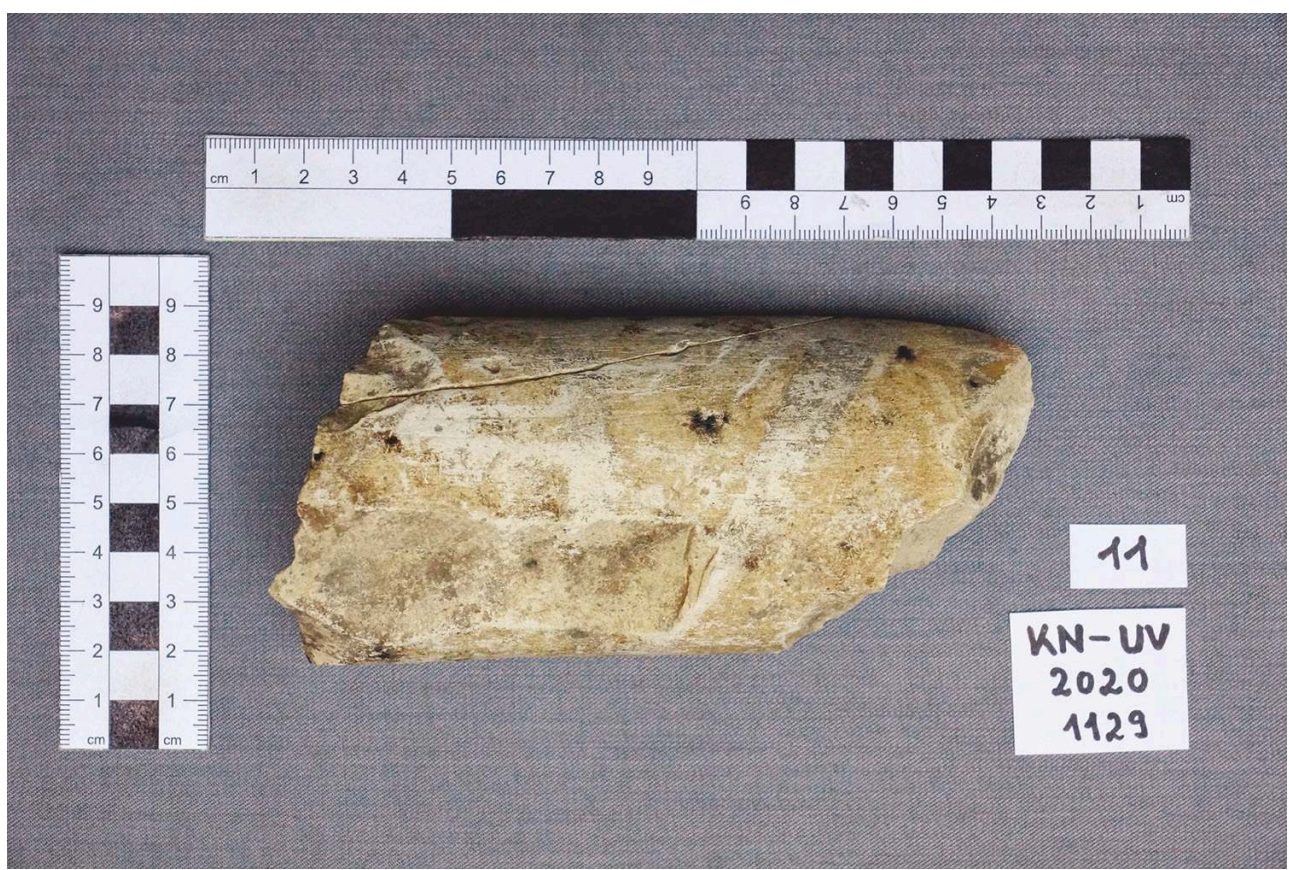

(C) Ifao. 17223_2020_NDMPM_007

\section{Petits objets}

Nous avons enregistré un total de 433 petits objets qui ne sont pas publiés à l'exception de quelques pièces. Ces objets sont faits de divers matériaux. Le groupe le plus nombreux est constitué de 257 objets en faïence (fig. 8-10): fragments d'amulettes, petites figurines, incrustations, carrelages, perles ou récipients. On a également dénombré 86 objets en argile, tels que des figurines d'animaux, des fermetures de pots ou des marques des potiers.

On a également enregistré 35 petits objets de pierres de types variés, tels que des pierres à polir, des perles, des récipients en pierre (fig. 11); 20 fragments : aiguilles en bronze, bagues, plaquettes; ainsi que quelques baguettes d'os et autres petits fragments divers.

21 La fig. 8 montre les jambes d'une petite figure masculine en faïence verte, d'environ $2 \mathrm{~cm}$ de haut. Les fig. 9 et 10 montrent la vue avant et arrière d'un fragment de corps d'ouchebti en faïence bleu-vert (H. environ $4 \mathrm{~cm}$ ). 
Fig. 8. Faïence verte, H. appr. 2 cm, inv. KNUV 20-2311 (photo-no. 2020-11086, G. Heumesser).

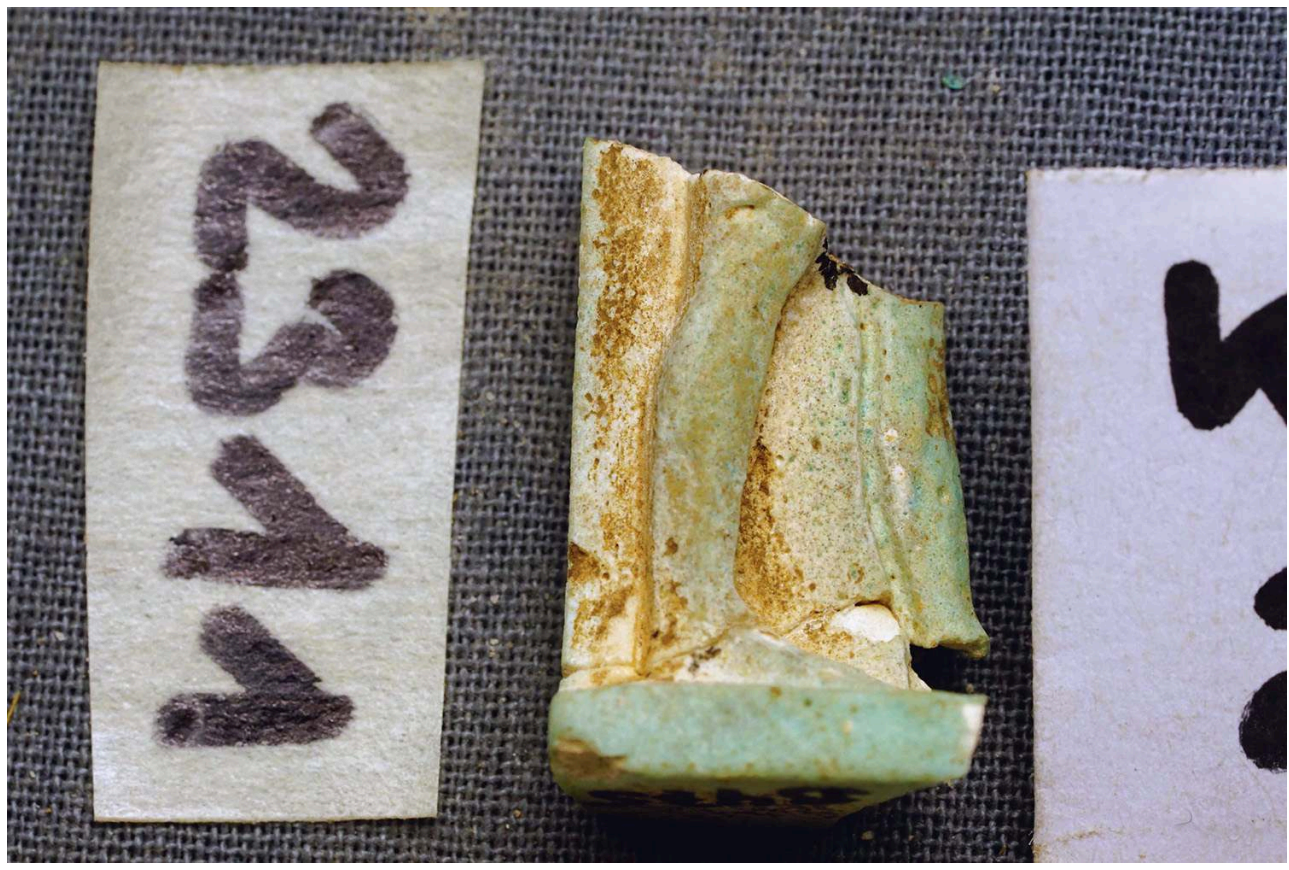

(c) Ifao. 17223_2020_NDMPM_008

Fig. 9. Torse d'ouchebti, front, faïence, inv. KNUV 20-2163 (photo-no. 2020_10741, G. Heumesser).

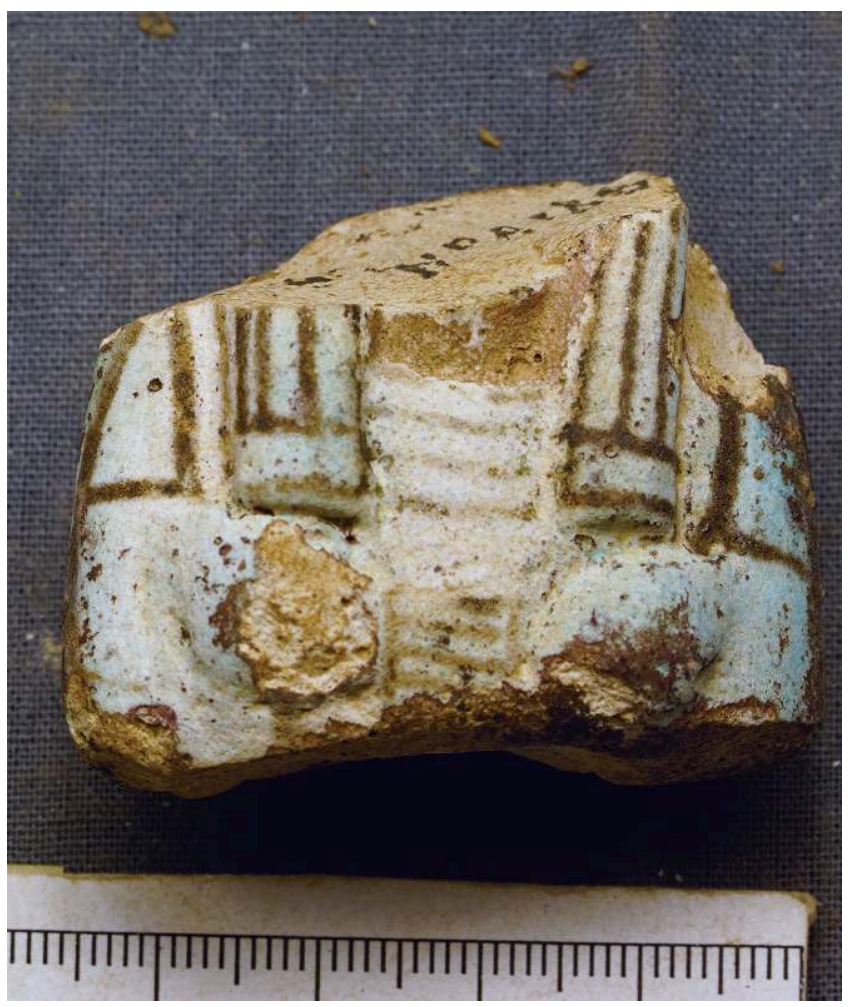

(c) Ifao. 17223_2020_NDMPM_009 
Fig. 10. Fragment d'épaules d'ouchebti. Verso, faïence, inv. KNUV 20-2163, (photo-no. 2020_10743, G. Heumesser).

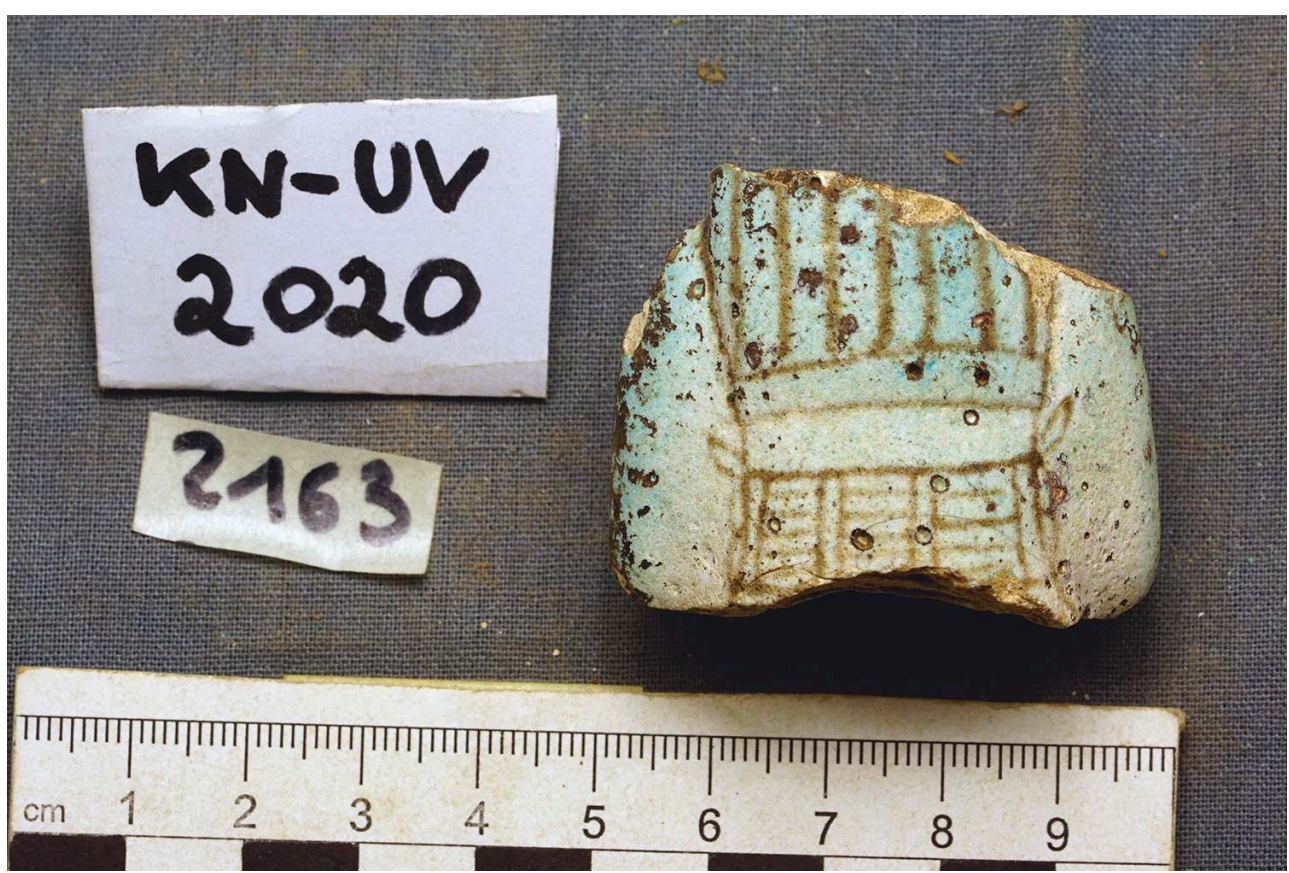

(C) Ifao. 17223_2020_NDMPM_010

Fig. 11. Fragment de vase en pierre, conique, calcite, inv. KNUV 20-2802, (photo-no. 2020_12575, G. Heumesser).

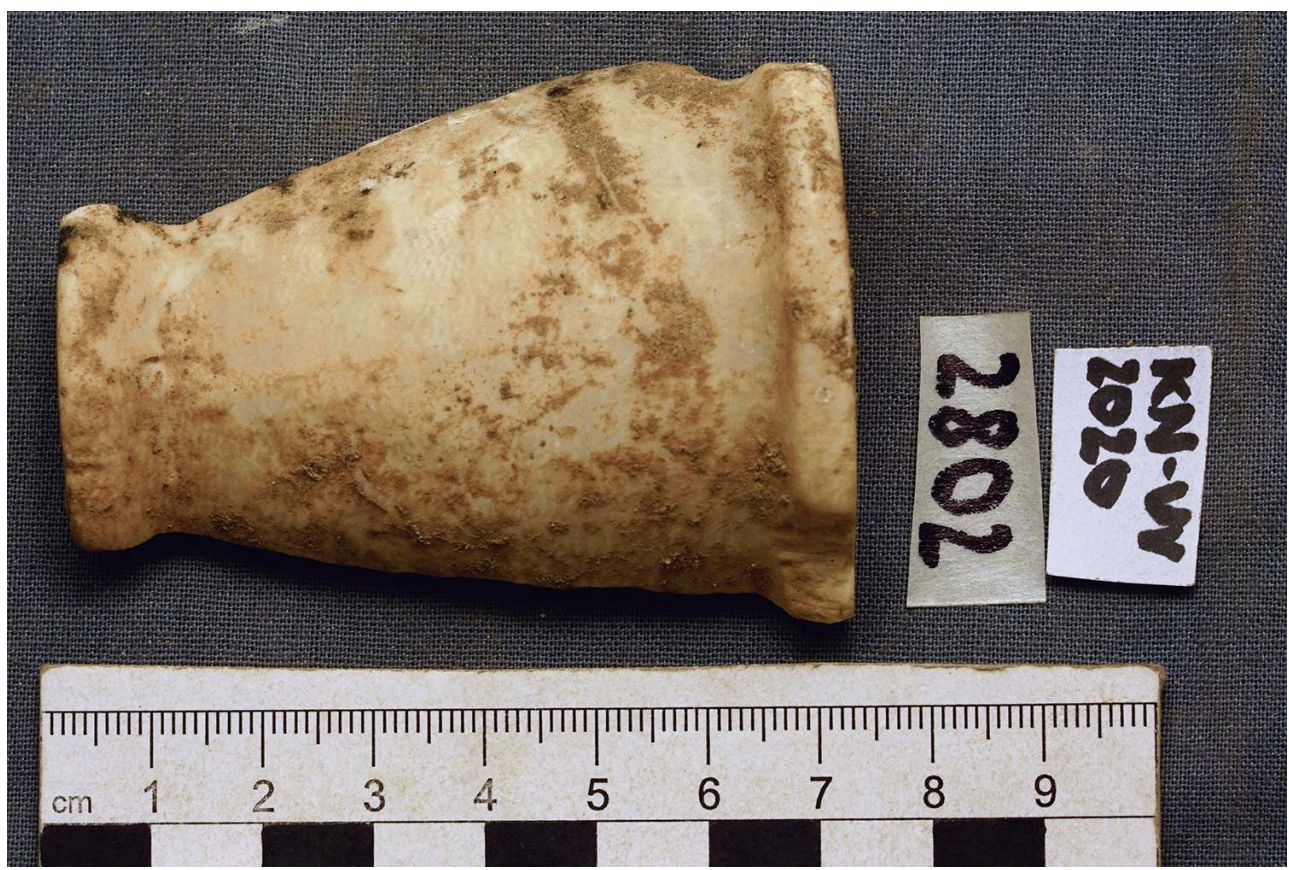

(c) Ifao. P17223_2020_NDMPM_017

22 Avec les 316 petits objets de 2019, nous avons maintenant enregistré 749 petits objets qui apparaîtront dans le catalogue.

Outre ces trois grands groupes d'objets (fragments de relief, fragments de pierre et petits objets), les modèles de pots d'offrandes trouvés dans des fosses de fondation, qui 
ont été publiés par Susan J. Allen ${ }^{7}$, ont également fait l'objet d'une documentation photographique.

Le graphique final de ce rapport offre un aperçu du nombre des fragments de reliefs enregistrés entre 2018 et 2020, et montre l'ampleur de la documentation photographique, qui contient également d'autres objets tels que des fragments de pierre, les petits objets ou les céramiques.

Fig. 12. Illustration du nombre de fragments de relief et de photographies des dépôts du Trésor de Thoutmosis ${ }^{\mathrm{er}}$, à partir de 2020 (graphique I. Hein).

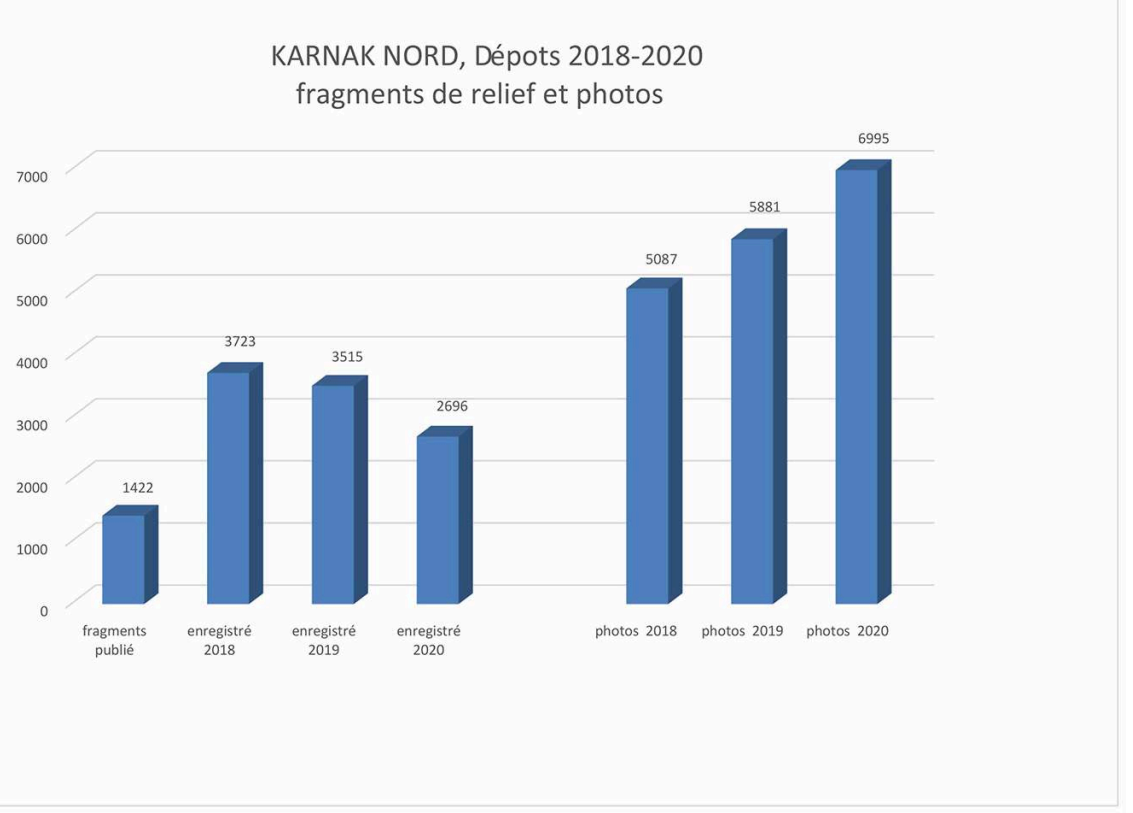

\section{BIBLIOGRAPHIE}

ALLEN 2012

Susan J. Allen, « Excursus B. Les ensembles de petits pots d'offrande trouvé dans la couche de démolition du trésor ", in Helen Jacquet-Gordon, Karnak-Nord X. Le Trésor de Thoutmosis Ir : la céramique, vol. 1, FIFAO 65, Le Caire, Ifao, 2012, p. 209-224.

HEIN 2020

Irmgard Hein, « Karnak-Nord - Storerooms », Bulletin archéologique des Écoles françaises à l'étranger, article en ligne sur OpenEdition Journals, http://journals.openedition.org/baefe/1158, consulté le 17 mai 2021.

JACQUET-GORDON 1988

Helen Jacquet-Gordon, Karnak-Nord VI. Le Trésor de Thoutmosis I ${ }^{e r}$ : la décoration, 2 vol., FIFAO 32, Le Caire, Ifao, 1988. 
JACQUET-GORDON 1999

Helen Jacquet-Gordon, Karnak-Nord VIII. Le Trésor de Thoutmosis I ${ }^{e r}$ : statues, stèles et blocs réutilisés, FIFAO 39, Le Caire, Ifao, 1999.

VARILLE 1943

Alexandre Varille, Karnak I, FIFAO 19, Le Caire, Ifao, 1943.

\section{NOTES}

1. JACQUET-GORDON 1988, p. 92 et pl. XXIII.

2. Cf. HEIN 2020.

3. JACQUET-GORDON 1988, p. 88.

4. JACQUET-GORDON 1999, p. 300, cat. n 190, fig. 206.

5. VARILLE 1943, p. 30, pl. LXXXVIII.C.

6. JACQUET-GORDON 1999, p. 281-283. cat. nº 181, fig. 194-196.

7. ALLEN 2012.

INDEX

Thèmes : IFAO

Année de l'opération : 2020

\section{AUTEURS}

\section{IRMGARD HEIN}

Égyptologue, Universität Wien 